\title{
Deep Learning and Analyses of Clustering Algorithms
}

\author{
Yang Li \\ School of Electronic Engineering, Xidian University, Xi'an, 710126, China
}

\begin{abstract}
The research actuality and new progress in clustering algorithm in recent years are summarized in this paper. First, the analysis and induction of some representative clustering algorithms have been made from several aspects, such as the ideas of algorithm, key technology, advantage and disadvantage. On the other hand, several typical clustering algorithms an d known data sets are selected simulation experiments are implemented from both sides of accuracy and running efficiency, and clustering condition of one algorithm with different data sets is an analyzed by comparing with the same clustering of the data set under different algorithms. Finally, the research hotspot, difficulty shortage of the data clustering and some pending problems are addressed by the integration of the aforementioned two aspects information. The above work can give a valuable reference for data clustering and data mining .
\end{abstract}

Keywords: Clustering algorithm, k-means, Data sets, Refining initial points, Cluster

\section{Introduction}

Clustering algorithm has been researched for decades, at the same time, clustering is indispensable to research of data mining and pattern recognition. For pattern recognition, clustering mainly used as speech recognition and character recognition. In machine learning, clustering algorithm is applied to image segmentation and machine vision. For image processing, clustering algorithm is applied to data compression and message retrieval, and another important usage of clustering is to apply into data mining, sequence, heterogeneous data analyses, etc.

In this paper, we analyzed clustering algorithm representatively that proposed in recent years about algorithm ideas, key technologies, advantages and disadvantages. And we choose some well-known data sets in experiments, after that, we made conclusion in terms of the analyses ${ }^{[1-3]}$.

Section 1 is introduction of clustering, cluster process and algorithms; section 2 focuses on seventeen representative algorithms; section 3 described eight results of simulation about clustering algorithm, and combined reference [4] for analyses; section 4 is conclusion.

\section{Clustering and categories of Algorithm}

\subsection{Concept of Clustering and Cluster Process}

There is no accepted definition of clustering in academia so far. In this paper, we show one definition mentioned by Everitt $^{[5]}$ in 1974: Entities in the same class clusters are analogous, entities in different class clusters are dissimilar;
A class cluster is the convergence of the midpoint of the test space, Distance between any two points of the same class cluster is less than that in different class cluster; Class cluster can be described as a regional connectivity which contain high density point set in multidimensional space, they are separated by low-density point set area or other area ( class cluster )

As the matter of fact, clustering is an unsupervised classification, and it has no prior knowledge can be used. The following is description of clustering:

Assume we have a set $U=\left\{p_{1}, p_{2}, \ldots, p_{n}\right\}$ represents a model set, where $p_{i}$ is the $i$-th model, $i=\{1,2, \ldots, n\} ; C t \subseteq U$, $\mathrm{t}=1,2, \ldots, \mathrm{k}, C_{t}=\left\{p_{t 1}, p_{t 2}, \ldots, p_{t w}\right\} ; \operatorname{proximity}\left(p_{m x}, p_{i r}\right) ;$ among them, the first subscript indicates the class which the pattern belongs to; the second subscript indicates a certain mode; function proximity is used to describe similarity distance of patterns. If $C_{t}$ is the result of clustering, it satisfies these following conditions:<smiles>C=CC</smiles>

2) For $\forall C_{m}, C_{r} \subseteq U, c_{m} \neq C_{r}$, and $C_{m} \cap C_{r}=\phi$ (Limited to rigid clustering)

$$
J(W, P)=\sum_{i=1, k}\left[\sum_{j=1,-n} w_{i j}^{2} \sum_{m=1,-, t} \lambda_{m}^{r}\left|x_{j m}^{r}-p_{j m}^{r}\right|^{2}+\sum_{j-1, n} w_{i j}^{2} \sum_{q-t+1,-m} \lambda_{q}^{c} \delta\left(x_{j q}^{c}, x_{j q}^{c}\right)\right]
$$

Typical clustering processes include data preparation, feature selection and extraction, proximity calculating, clustering (Grouping), making effectively evaluation of clustering results ${ }^{[3,6,7]}$.
Clustering process:

1) Data Preparation: Standardize features and reduce dimensionality.

2) Feature Selection: Choose the most effective feature 


\section{International Journal of Science and Research (IJSR) \\ ISSN (Online): 2319-7064}

Index Copernicus Value (2013): 6.14 | Impact Factor (2014): 5.611

from the initial feature, and store it into vector.

3) Feature Extraction: Transform the feature which was selected, in order to shape a new prominent feature.

4) Clustering(Grouping): Choosing a suitable characteristic distance function, measure its closeness, then clustering or grouping.

5) Evaluation of clustering results: Evaluation of external validity; Evaluation of inner validity; Evaluation of the text of correlation.

\subsection{Category of Clustering Algorithm}

There is no clustering algorithms could be generally used to reveal various structures appeared from multidimensional data aggregation. Clustering algorithm includes several classifications, in this paper, we classify this algorithm into hierarchical clustering algorithm, disconnected clustering algorithm, clustering algorithm based on density and grid, and other clustering algorithm.

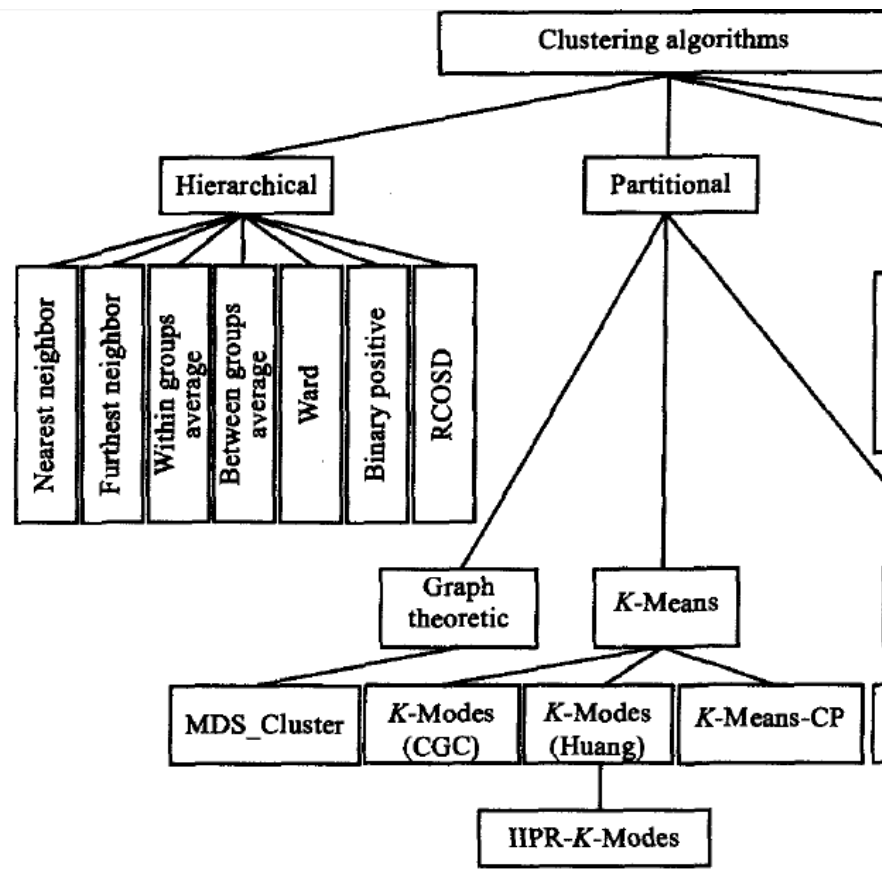

Figure 1: The classification chart of clustering algorithms

\section{Clustering Algorithms}

\subsection{Hierarchical clustering algorithm}

Hierarchical clustering algorithm is called Trees clustering algorithm $^{[8,9]}$. It repeatedly retrievals or clusters data by means of hierarchical structure, in order to form a clustering answer to hierarchical sequence. The complexity of computation is $O\left(n^{2}\right)$, this algorithm is used in classification of small data sets.

Assume a sample set $S=\left\{O_{1}, O_{2}, \ldots, O_{n}\right\}$ has $n$ samples in all

HA1[Initialization]. Regard every sample $o_{r}$ as a class;

$/ *$ form $n$ classes in all: $\quad o_{l}, O_{2}, \ldots, O_{n} * /$

HA2[Figure out two nearest classes].

$\operatorname{dis} \tan c e\left(o_{r}, o_{k}\right)=\min _{\forall o_{u}, o_{v} \in S, o_{u} \neq o_{v}} \operatorname{dis} \tan c e\left(o_{u}, o_{v}\right)$;
$/ *$ Locate the nearest two classes from all classes $o_{r}$ and $o_{k}^{*} /$

HA3[Cluster $o_{r}$ and $o_{k}$ ]. Then we get a new class $o_{r k}$; $/ *$ Existing class will be reduced by $1 * /$

HA4. If all the samples belong to a same class, end this algorithm; otherwise, back to HA2.

\subsection{Traditional clustering rules}

Methods of measure the distance between two classes is one important parts of traditional hierarchical aggregation algorithm. In this paper, we use Euclidean distance to measure similarity. Connection rules contain single connection rules, fully connection rules, average connection between class rules, average connection within one class rules, and Ward rules ${ }^{[8]}$ (where $\|x-y\|$ is Euclidean norm, $n_{i}$ and $n_{k}$ are the numbers of samples in class $o_{r}$ and $o_{k}$, total methods of two different elements chosen from $n_{i}+n_{k}$ denoted as $\left(+n_{1}+n_{k, 2}\right)$ );

\section{PiBgensify, gnigction clyusteringtheles:}

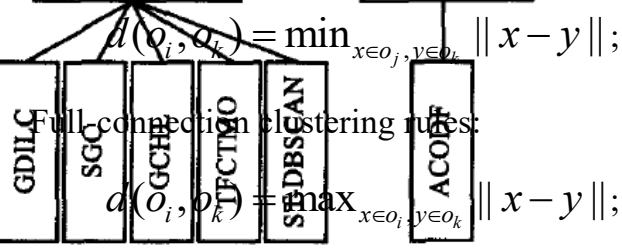

Average-connection between multiple classes rules:

$\mathrm{FCM}_{\mathrm{F}} \mathrm{dt}\left(\rho_{i}, o_{k}\right)=\left(1 / n_{i} n_{k}\right) \sum_{x \in o_{i}}\left(\sum_{y \in o_{k}}\|x-y\|\right)$;

Average-connection within one class rules:

NFWFCA $p_{i}^{\text {FGFGM }}=\left(1 / C\left(n_{i}+n_{k}, 2\right)\right) \sum_{x, y \in\left(o_{i}, o_{k}\right)}\|x-y\|$;

Ward

$$
\text { rules: } d\left(o_{i}, o_{k}\right)=\left(1 /\left(n_{i}+n_{k}\right)\right) \sum_{x \in\left(o_{i}, o_{k}\right)}\|x-n\|^{2} \text {, }
$$

where $n$ is the center of fusion clustering.

\subsection{New Hierarchical clustering algorithm}

(1) Binary-Positive

In 2007, Glebard ${ }^{[4]}$ et al. suggested a new hierarchical clustering algorithm, which is called Binary-Positive. There are many methods represented in Dice to measure various Binary-Positive similarity ${ }^{[10,11]}$

Glebard et al. adopted these four data sets: Wine, Iris, Ecolic and Psychology balance, to experiment between eleven kinds of clustering algorithms. The results show that all the algorithms are well-used in experiments.

(2) Rough clustering of sequential data(RCOSD)

In 2007, Kumar ${ }^{[12]}$ et al. argued a new hierarchical clustering algorithm based on indistinguishable crude aggregation: RCOSD. This algorithm introduce $S^{3} M$ as a way to measure similarity. This algorithm could merger more than two classes every time, so it could accelerate the

\section{Volume 4 Issue 12, December 2015}




\section{International Journal of Science and Research (IJSR) \\ ISSN (Online): 2319-7064}

Index Copernicus Value (2013): 6.14 | Impact Factor (2014): 5.611

speed of hierarchical aggregation.

The results show that RCOSD is available, this algorithm could help Webers discriminate potentially significant user groups.

\subsection{Partition clustering algorithm}

Partition clustering algorithm should specify the number of clusters or center of clusters in advance. By using duplicate iteration calculations, gradually reduce the error of the objective function. When the value of the objective function converges, we get final result of clustering.

\subsection{1 $K$-means clustering algorithm}

In 1967, Mac Queen mentioned $K$-means clustering algorithm for the first time, so far, several clustering missions have chosen this classical algorithm. The main idea of this algorithm is to find out $K$ clustering centers: $c_{l}$, $c_{2}, \ldots, c_{k}$, in order to minimize every data points $x_{i}$ and the sum of square of the distance of the closest clustering center $c_{v}$ (This sum of square of the distance is called deviation $D$ ). $K$-means clustering algorithm ${ }^{[8]}$ ( clustering of $n$ samples)

K1[Initialization]. Assign $K \quad$ clustering centers randomly $\left(c_{1}, c_{2}, \ldots, c_{K}\right)$;

K2[Assign $x_{i}$ ]. For every sample $x_{i}$, find the closest clustering center $c_{v}$, and assign $x_{i}$ into class $c_{v}$ that indicated; $\mathbf{K} 3\left[\right.$ Correct $c_{w}$ ]. Put every $c_{w}$ into indicated class center;

K4[Calculate deviation].

$D=\sum_{i=1}^{n}\left[\min _{r=1, \ldots K} d\left(x_{i}, c_{r}\right)^{2}\right]$

$\mathbf{K 5}[D$ is convergent or not?]. If $D$ is convergent, then return $\left(c_{1}, c_{2}, \ldots, c_{K}\right)$ and end this algorithm; Otherwise, back to $\mathbf{K} 2$.

Advantage: $K$-means clustering algorithm could classify large-scale dataset efficiently, and this algorithm calculates faster than Hierarchical clustering algorithm.

\subsection{2 $\mathrm{K}$-modes algorithm}

(1) $K$-modes-Huang algorithm ${ }^{[14]}$

Introduction of Means and Modes:

In $K$-means algorithm, mean is center of clusters, and can be defined randomly at first. In $K$-modes algorithm, mode is defined as: data set $X=\left\{X_{1}, X_{2}, \ldots X_{n},\right\}, \forall X_{i} \in X$ described by $m$ categorical attributes $\left\{A_{1}, A_{2}, \ldots A_{m}\right\}, X_{i}$ is denoted as vector $<x_{i 1}, x_{i 2}, \ldots, x_{i m}>$; $Q$ is one of the modes $X, Q$ is denoted as vector $\left\langle q_{1}, q_{2}, \ldots, q_{m}>\right.$, and $Q$ satisfies $\sum_{i=1 \ldots . . n} d_{1}\left(X_{i}, Q\right)$ minimum. $d_{1}\left(X_{i}, Q\right)$ is distance between $X_{i} \ldots$ and $Q$.

Same as $K$-means algorithm, $K$-modes algorithm is also able to produce locally optimal solution, relying on the choice of modes initialization and sequence of centralized data.

In 1999, Huang ${ }^{[15]}$ et al. made the conclusion $K$-means algorithm can only converge local minimum.
(2) $K$-modes-CGC algorithm ${ }^{[16]}$

In 2001, Chaturvedi et al. suggested a new nonparametric clustering method, which was called $K$-modes-CGC algorithm. This algorithm is similar to traditional $K$-means algorithm(the prior algorithm). $K$-modes-CGC algorithm optimizes one loss function based on norm $L_{0}$.

In Monte Carlo simulation, Chaturvedi et al. used $K$-modes-CGC ${ }^{[17]}$ and latent class algorithm to restore a known latent class structure. The results show that, both algorithm have equivalent efficiency.

In 2003, Huang ${ }^{[18]}$ et al. proved that $K$-modes-CGC algorithm is equivalent to $K$-modes-Huang algorithm.

\subsubsection{Refining initial points for $K$-modes}

In 2002, Sun $^{[19]}$ et al. applied refining initial points for $K$-means suggested by Bradley ${ }^{[20]}$ into $K$-modes(Huang,1998). Sun et al. proposed an experiment based on refining initial points for $K$-modes.

Sun's experiment based on well-known soybean disease dataset. Data of soybean disease contain 47 records, every record was described by 35 characteristics, and every record was signed one of the following four diseases: Diaporthe StemCanker, Charcoal Rot, Rihizoctonia Root Rot, and Phytophthora Rot. Apart from Phytophthora Rot has seventeen records, other three diseases have ten records. There are two programs about $K$-modes:

Program 1: Randomly select initial point sets;

Program 2: Use refining initial points for $K$-modes to select initial point sets.

In addition, program 2 produces precision reliable clustering resultss.

\subsection{4 $K$-means Consistency Preservation algorithm} (K-means-CP)

In 2004, Ding ${ }^{[22]}$ et al. put forward $K$-means Consistency Preservation algorithm. Nearest neighbor consistency is a important concept in statistical pattern recognition, they expended this concept into data clustering: As any data points in one class, its nearest neighbor and mutual nearest neighbor of $k$ should belong to this class. They proposed improved algorithm for $k \mathrm{NN}$ and $k \mathrm{MN}$, and regarded the nearest neighbor and mutual nearest neighbor of $k$ as an important way of metrics.

$K$-means-CP:

1 [Initialization]. Choose $K$ points as centers of the initial class randomly $\left(c_{1}, c_{2}, \ldots, c_{K}\right)$

2[Assign neighbor sets]. Assign a neighbor set $S$; //*Assign $S$ into nearest class $C_{p}$,

$$
p=\arg \min _{v=1, \ldots, K} \sum_{x_{i} \in S}\left(x_{i}-m_{v}\right)^{2}
$$

$3\left[\right.$ Update clustering center]. Define $m_{v}=\sum_{x_{i} \in C_{v}} x_{i} / n_{v}$;

//* Update cluster center (centroid), $m_{v}$ is center of class $C_{v}$, $n_{k}=\left|C_{k}\right|$ 


\section{International Journal of Science and Research (IJSR) \\ ISSN (Online): 2319-7064}

Index Copernicus Value (2013): 6.14 | Impact Factor (2014): 5.611

$4[$ Convergent or not?]. If centroid no longer move, then end the algorithm; Otherwise, back to Step2. $/ / * J_{K m}=\sum_{v=1, \ldots, K} \sum_{x_{i} \in C_{v}}\left(x_{i}-m_{v}\right)^{2}$ and judge it is convergant or not.

\subsubsection{Fuzzy clustering algorithm}

In 1969, Ruspini applied fuzzy sets theory into clustering analyses for the first time, and proposed fuzzy clustering means(FCM). FCM is one of the most popular algorithms dealing with image segmentation. FCM can retain more information of initial images. In this paper, we simply introduce the newest study ${ }^{[23,24]}$.

In $2006, \mathrm{Li} \mathrm{Jie}^{[25]}$ et al. proposed new algorithm NFWFCA based on feature weighting. Traditional fuzzy $K$-means, $K$-modes, and $K$-prototype all assume that contributions of

$$
J(W, P)=\sum_{i=1, \ldots, k}\left[\sum_{j=1, \ldots, n} w_{i j}^{2} \sum_{m=1, \ldots, t} \lambda_{m}^{r}\left|x_{j m}^{r}-p_{j m}^{r}\right|^{2}+\sum_{j=1, \ldots, n} w_{i j}^{2} \sum_{q=t+1, \ldots, m} \lambda_{q}^{c} \delta\left(x_{j q}^{c}, x_{j q}^{c}\right)\right]
$$

When $J(W, P)$ is the minimum, the result of clustering is optimal. When $\lambda^{c}=0$, it corresponds $K$-means; when $\lambda^{r}=0$, it corresponds $K$-modes; when $\lambda^{c} ¥ 0$, it corresponds fuzzy $K$ - prototype.

The results show that this new algorithm is more efficient and accurate. This makes great progress in clustering algorithm researches.

In $2007, \mathrm{Cai}^{[27]}$ et al. combined with local spatial and gray information, proposed clustering algorithm FGFCM based on FCM, characterized: (1) Use a new factor $S_{i j}$ as partial(space and gray) similarity measurement. Not only keep immunity of image, retain image details, but also expect adjustable parameter $\alpha$; (2) Split time only relate to gray level $q$, its complexity reduce from $O\left(\mathrm{NcI}_{l}\right)$ to $O\left(q c I_{2}\right)$, where $c$ is clustering number, $I_{l}$ and $I_{2}\left(<I_{l}\right)$ are iterations of FCM and FGFCM; (3) FGFCM can be used for many other algorithms, FCM, EnFCM, FGFCM_S1 and FGFCM_S2 all can be deduced.

\subsubsection{Graph Theory Algorithm}

In 1999, Jain ${ }^{[3]}$ suggested famous graph theory fission clustering algorithm: Construct a minimal spanning tree(MST) based on datas, by deleting the longest leg of minimal spanning tree. The algorithms based on graph theory include Random Walk, CHANMELEON, AUTOCLUST $^{[28,29,30,31]}$.

In 2007, $\mathrm{Li}^{[31]}$ suggested a clustering algorithm based on maximum $\theta$ distance subtree $\theta$ denoted as MDS CLUSTER. Cut of all edges which length greater than the threshold $\theta \geq 0$, generating maximum $\theta$ distance subtree sets, and vertexes of every maximum $\theta$ distance subtree make up one class. dimensional feature for each sample vector are the same. In fact, contributions of dimensional feature for each sample vector are discriminate. Based on $K$ - prototype, NFWFCA used ReliefF ${ }^{[26]}$ algorithm in order to determine weight of each dimensional feature:

$$
\lambda^{r}=\lambda^{r}-\frac{\text { diff }_{-} \text {hit }^{r}}{R}+\frac{\text { diff }_{-} \text {miss }^{r}}{R}
$$

Property feature weights compute as:

$$
\lambda^{c}=\lambda^{c}-\frac{\text { diff }_{-} \text {hit }^{c}}{R}+\frac{\text { diff }_{-} \text {miss }^{c}}{R}
$$

Revise the object function:

\section{Clustering Algorithm based on gird and density}

Clustering algorithm based on gird and density is one important clustering algorithm, and it is widely used in many sphere especially in spatial information processing.

Different from traditional clustering algorithm, this algorithm could discover arbitrary shape clustering by using data density; this clustering algorithm familiarly combine with other algorithms, especially clustering algorithm based on density.

In 2001, Zhao and Song ${ }^{[32]}$ mentioned a clustering algorithm GDILC which based on grid density contours. The main idea of GDILC is: use density contours image to depict data sample distribution, and use network to calculate each density of data sample. The results show that GDILC has high accuracy and efficient characteristic.

In $2004, \mathrm{Ma}^{[33]}$ proposed a new algorithm $\mathrm{SGC}$ which based on shifting grid concept. SGC is a non-parametric algorithm, it does not need users to input parameters, for it divide every latitude of grid structures into one data space. SGC produces displacement concept of whole grid structures, hence, could improve results accurate and efficient.

In 2005, Pileva ${ }^{[34]}$ et al. advanced gird clustering algorithm(GCHL) base on large, high-dimensional database. GCHL combines density-gird clustering algorithm with parallel shaft partitioning strategy, in order to make sure the high density region. This algorithm can be used excellently in random spatial database.

In 2006, Micro $^{[35]}$ et al. faced to moving object trajectory data processing sphere, in view of simple concept of distance between the track, put forward an adaptive clustering algorithm base on density(TFCTMO). 


\section{International Journal of Science and Research (IJSR) \\ ISSN (Online): 2319-7064 \\ Index Copernicus Value (2013): 6.14 | Impact Factor (2014): 5.611}

In 2007, Derya ${ }^{[36]}$ et al. expanded DBSCAN(density-based spatial clustering of applications with noise), and then proposed a new algorithm based on density which was called ST-DBSCAN(spatial-temporal DBSCAN), comparing with existing clustering algorithm based on density, ST-DBSCAN has ability in discovering class clusters relying on non-space value, space value, and tense value.

\subsection{Other clustering algorithms}

\subsubsection{ACODF clustering algorithm}

In 2004, Tsai ${ }^{[37]}$ proposed a novel with different preferences ant colony system(novel AS)-ACODF(a novel data clustering approach for data mining in large databases), in order to solve clustering problems. ACODF is able to obtain optimal solution quickly, it contain three important strategies as following:

1) The application of different preferences(favorable) ACO strategy. Each ant only visit one tenth of all the cities, then successively reduce number of cities each visit; After several cycles, the concentration of pheromone increased between two closer points, and the concentration of pheromone reduced between two distant points. Therefore, ants would like to visit closer nodes, and use pheromones to strengthen this path, finally, form a high concentration path, then clustering completed.

2) We design two formulas:

$$
n s(t+1)=n s(t) \times T
$$

where $n s$ is the number of nodes that ants visit in $T_{0} ; n s(t+1)$ is the number of nodes that ants have been visited yet; $n s(t)$ is the number of nodes that ants visited in the last cycle; $T$ is a constant $(T=0.95)$.

$$
n f(t+1)=2 \times n s(t) / 3-i \times n s(t) /(r u n \times 3)
$$

where $n f$ is the number of nodes that ants visit in $T_{1} ; n f(t+1)$ is the number of nodes that ants have been visited yet; $n f(t)$ is the number of nodes that ants visited in the last cycle; run $=2, i \in\{1,2\}$.

(1) Using tournament selection strategy. Different form traditional ACO, ACODF uses tournament selection strategy in order to select path. That is select $K$ paths randomly in $N$ paths, then choose the shortest path in these $K$ paths $(N>K)$.

\section{Experiments}

In this paper, we choose five data sets: Iris, Wine, Soybean, Zoo and Image. Image data set is used to compared with Iris and Wine datasets.

For numerical model data, respectively use Iris, Wine, Image for experiments.

Iris includes 3 classes, each class has 50 elements, and every class represent one kind of flowers, 150 samples-equidistribution in 3 clusters; among them, one class of linear separable with other two class, and other two class are partially overlapped. Data set Wine has good clustering structure, including 178 samples, 13 numerical model property, divided into 3 classes, where contain different amount of samples. Image derived from UCI machine learning data sets, randomly chosen from 7 outdoor image sets.

For categorical attribute data, respectively used Soybean and Zoo dataset for experiments.

Dataset Soybean has 47 samples, including 35 attributes, divided into 4 classes for linear separable. Its attributes are all categorical attributes. Dataset Zoo has 101 records, divided into 7 classes for linear inseparable. In data set Zoo, 16 attributes used to describe samples, 15 of them are Boolean property value $\{0,1\}$ and 1 categorical attribute(leg count) $\{0,2,4,5,6,8\}$.

\subsection{Soybean disease data set experiment}

We use the formula of accuracy:

$$
r=\sum_{i=1, \ldots, k}\left(a_{i} / n\right)
$$

Where $a_{i}$ the sample number which emerges in the $i$-th cluster(obtain from this algorithm) and initial-points, $k$ is cluster number $(k=4), n$ is total samples number $(n=47)$.

Tab.1 and Tab.2 show the experiment result of this algorithm.

Tab.1 Clustering results of 20 random tests for soybean

\begin{tabular}{|c|c|c|}
\hline \multicolumn{3}{|c|}{ disease data set on 2 algorithms } \\
\hline Accuracy (\%) & $\begin{array}{c}\text { Iterative } \\
\text { initial-points } \\
\text { refinement } \\
K \text {-modes }\end{array}$ \\
\hline 98 & 5 & 7 \\
94 & 6 & 8 \\
77 & 0 & 3 \\
70 & 0 & 1 \\
68 & 7 & 1 \\
2 & 0 \\
\hline
\end{tabular}

Table 2: Average run time of 20 random tests for soybean disease data set on 2 algorithms

\begin{tabular}{|c|c|}
\hline Algorithm & Average running time(s) \\
\hline$K$-modes & 0.00817331 \\
\hline $\begin{array}{c}\text { Iterative initial-points } \\
\text { refinement } K \text {-modes }\end{array}$ & 0.01178265 \\
\hline
\end{tabular}

Basing on the running time, we can figure out that iterative initial-points refinement $K$-modes runs longer than $K$-modes algorithm. 


\section{International Journal of Science and Research (IJSR) \\ ISSN (Online): 2319-7064}

Index Copernicus Value (2013): 6.14 | Impact Factor (2014): 5.611

\subsection{Hierarchical clustering and $K$-means algorithm}

For numerical model data, we randomly did 20 cluster experiments using data set Iris, Wine, Image, and the results show in Tab.4.

Table 4: Clustering results of 20 random tests for Iris, Wine, Image data sets on several algorithms

\begin{tabular}{|l|r|c|c|c|c|c|}
\hline \multirow{2}{*}{ Algorithm } & \multicolumn{4}{|c|}{$\begin{array}{l}\text { Average accuracy of } \\
\text { running 20 cycles(\%) }\end{array}$} & \multicolumn{3}{l|}{ Average running time(s) } \\
\cline { 2 - 7 } & Inis & Wine & Image & Iris & Wine & Image \\
\hline Nearest neighbor & 68.00 & 42.70 & 30.00 & 1.5831 & 3.1346 & 5.2414 \\
Furthest neighbor & 84.00 & 67.40 & 39.00 & 1.5042 & 3.1434 & 5.6708 \\
Between groups average & 74.70 & 61.20 & 37.00 & 1.5027 & 3.1526 & 5.7853 \\
Wardmethod & 89.30 & 55.60 & 60.00 & 2.3793 & 4.7757 & 8.9599 \\
K-means & 81.60 & 87.96 & 56.00 & 0.0026 & 0.0038 & 0.0457 \\
\hline
\end{tabular}

The results show us, operating efficiency of these five clustering algorithms were significantly different between data sets. Thus, in a real world application, we should use different algorithms for different data sets question

\subsection{Comparison between $K$-means and $K$-means-CP algorithm}

In order to figure out whether $K$-means-CP is obviously better than $K$-means or not, and the relationship between $k \mathrm{NN}$ consistency and cluster quality, we did 20 random experiments on $K$-means, $1 K$-means- $\mathrm{CP}(k=1$,denoted as cp1), and $2 K$-means-CP $(k=2$,denoted as cp2), and evaluated the results based on accuracy and quality. The difference within one class, the difference between multiple classes in a whole cluster, and quality can be compute as following formula(1) (3):

$$
\begin{gathered}
\sum_{v=1, \ldots, k} \sum_{x \in C_{v}} d\left(x, \overline{x_{v}}\right)^{2} \\
\sum_{1 \leq j<i \leq k} d\left(\overline{x_{j}}, \overline{x_{i}}\right)^{2} \\
\sum_{1 \leq j<i \leq k} d\left(\overline{x_{j}}, \overline{x_{i}}\right)^{2} / \sum_{v=1, \ldots, k} \sum_{x \in C_{v}} d\left(x, \overline{x_{v}}\right)^{2}
\end{gathered}
$$

where $k$ is the number of cluster in clustering result, $C_{v}$ denotes cluster $v, \overline{x_{v}}$ denotes the centroid of $C_{v}, \overline{x_{j}}, \overline{x_{i}}$ respectively denote the centroid of cluster $j$ and $i, d$ is distance function. Tab. 6 shows the result that $K$-means-CP not better than $K$-means, $k \mathrm{NN}$ consistency has nothing to do with clustering quality.

\begin{tabular}{|c|c|c|}
\hline Imagine & $\begin{array}{c}\text { Average accuracy } \\
\text { (20 times) }\end{array}$ & $\begin{array}{c}\text { Average quality } \\
\text { (20 times) }\end{array}$ \\
\hline $\mathrm{cpl}(1 \mathrm{NN})$ & 0.623571428571428 & 0.7780367508393800 \\
\hline $\operatorname{cp} 2(2 \mathrm{NN})$ & 0.609523809523809 & 0.7647536177176110 \\
\hline$K$-means & 0.632380952380952 & 0.7340763582917190 \\
\hline Iris & $\begin{array}{c}\text { Average accuracy } \\
\text { (20 times) }\end{array}$ & $\begin{array}{l}\text { Average quality } \\
\text { (20 times) }\end{array}$ \\
\hline $\operatorname{cpl} 1(1 N N)$ & 0.840000000000000 & 0.2586261724481240 \\
\hline $\operatorname{cp} 2(2 \mathrm{NN})$ & 0.892333333333333 & 0.3224891574120460 \\
\hline$K$-means & 0.862333333333334 & 0.2902686923643110 \\
\hline Wine & $\begin{array}{l}\text { Average accuracy } \\
\text { (20 times) }\end{array}$ & $\begin{array}{c}\text { Average quality } \\
\text { (20 times) }\end{array}$ \\
\hline $\operatorname{cpl}(1 \mathrm{NN})$ & 0.898314606741573 & 0.0454332393240636 \\
\hline $\operatorname{cp} 2(2 \mathrm{NN})$ & 0.905337078651385 & 0.0451553609767059 \\
\hline$K$-means & 0.946910112359550 & 0.0490987358800575 \\
\hline Glass & $\begin{array}{c}\text { Average accuracy } \\
\text { (20 times) }\end{array}$ & $\begin{array}{c}\text { Average quality } \\
\text { (20 times) }\end{array}$ \\
\hline $\operatorname{cpl}(1 \mathrm{NN})$ & 0.511915887850467 & 0.400881509658679 \\
\hline $\operatorname{cp} 2(2 \mathrm{NN})$ & 0.531542056074766 & 0.404061886906006 \\
\hline$K$-means & 0.542523364485981 & 0.453522047430905 \\
\hline Ionosphere & $\begin{array}{c}\text { Average accuracy } \\
\text { (20 times) }\end{array}$ & $\begin{array}{c}\text { Average quality } \\
\text { (20 times) }\end{array}$ \\
\hline $\operatorname{cp} 1(1 \mathrm{NN})$ & 0.691880341880342 & 0.0038124768513412 \\
\hline $\mathrm{cp} 2(2 \mathrm{NN})$ & 0.682051282051282 & 0.0035553114620347 \\
\hline$K$-means & 0.710256410256410 & 0.0037845994509161 \\
\hline
\end{tabular}

Table 6: Clustering results of 20 random tests for 5 data sets on $K$-means, cp1\&cp2 algorithm

\section{Conclusion}

By means of experiments for several clustering algorithm, we can figure out most clustering algorithms need prescribed parameters. Thus, promoting non-prescribed parameters clustering algorithm, combining clustering algorithm with parameters autogeneration algorithm may have good prospect. And RCOSD can efficiently work in

\section{Volume 4 Issue 12, December 2015}




\section{International Journal of Science and Research (IJSR) \\ ISSN (Online): 2319-7064}

Index Copernicus Value (2013): 6.14 | Impact Factor (2014): 5.611

data mining, helping us to understand the results of clustering.

The algorithms discussed in this paper apply to data sets with different properties, accordingly, researchers should use different algorithms and methods for divergent data problems. As a supplement for above conclusion, we compared 11 different algorithms ${ }^{[4]}$ and 8 algorithms proposed in this paper. Tab. 7 shows the comparative results of some typical clustering algorithms.

Table 7: Comparative results of several typical clustering algorithms

\begin{tabular}{|c|c|c|c|c|c|c|c|c|}
\hline Algorithm & Years & Sort & $\begin{array}{l}\text { Similarity } \\
\text { measure }\end{array}$ & Parameter & Noise & $\begin{array}{l}\text { Cluster } \\
\text { shape }\end{array}$ & $\begin{array}{c}\text { Scaled } \\
\text { dimension }\end{array}$ & Others \\
\hline$K$-means & 1967 & Partition & $\begin{array}{l}\text { Distance } \\
\text { function }\end{array}$ & 1 & Sensitive $\mathrm{H}$ & Hypersphere & $\begin{array}{r}\text { Large } \\
\text { numeric }\end{array}$ & - \\
\hline$K$-means-Huang & 1998 & Partition & $\begin{array}{l}\text { Category } \\
\text { similarity } \\
\text { function }\end{array}$ & 1 & Sensitive & Sphere & $\begin{array}{l}\text { Large } \quad \mathrm{D} \\
\text { category }\end{array}$ & $\begin{array}{l}\text { escribe cluster } \\
\text { well }\end{array}$ \\
\hline$K$-means-CP & 2004 & Partition & $\begin{array}{l}\text { Distance } \\
\text { function }\end{array}$ & 1 & Sensitive & Sphere & $\begin{array}{r}\text { arge-Scale } k \mathrm{~N} \\
\text { is } \\
\text { cluster }\end{array}$ & $\begin{array}{l}\text { N consistency } \\
\text { irrelevant with } \\
\text { ring accuracy }\end{array}$ \\
\hline MDS_CLUSTER & 1998 & Partition & $\begin{array}{l}\text { Eulidean } \\
\text { distance }\end{array}$ & 1 & In-Sensitive & $\begin{array}{l}\text { Arbitrary } \\
\text { non-overlap }\end{array}$ & - & $\begin{array}{l}\text { One simple } \\
\text { parameter }\end{array}$ \\
\hline $\begin{array}{l}\text { Feature weighted } \\
\text { fuzzy clustering }\end{array}$ & 2004 & Partition & $\begin{array}{l}\text { Eulidean distance } \\
\text { category similarit } \\
\text { measure }\end{array}$ & ty & In-Sensitive & Sphere & Small,mix & $\begin{array}{l}\text { Feature } \\
\text { weighted }\end{array}$ \\
\hline Nearest neighbor & 1967 & Hierarchy & $\begin{array}{l}\text { Distance } \\
\text { function }\end{array}$ & 1 & In-Sensitive & Filamentary & $\begin{array}{l}\text { Small and } \\
\text { middlow- } \\
\text { dimension }\end{array}$ & - \\
\hline Furthestneighbor & 1967 & Hierarchy & $\begin{array}{l}\text { Distance } \\
\text { function }\end{array}$ & 1 & - & Sphere & $\begin{array}{l}\text { Small and } \\
\text { middlow- } \\
\text { dimension }\end{array}$ & - \\
\hline $\begin{array}{c}\text { Between groups } \\
\text { average }\end{array}$ & 1967 & Hierarchy & $\begin{array}{l}\text { Distance } \\
\text { function }\end{array}$ & 1 & - & Manifold & $\begin{array}{l}\text { Small and } \\
\text { middlow- } \\
\text { dimension }\end{array}$ & - \\
\hline $\begin{array}{l}\text { Sequence data } \\
\text { rough clustering }\end{array}$ & 2007 & Hierarchy & $S^{a} M$ & 2 & - & Sequence data & Large-Scale & $\begin{array}{c}\text { Depict } \\
\text { cluster feature }\end{array}$ \\
\hline SGC & 2004 & Density & $\begin{array}{l}\text { Distance } \\
\text { function }\end{array}$ & None & In-Sensitive & $\begin{array}{c}\text { Arbitrary } \\
\text { shape }\end{array}$ & $\begin{array}{l}\text { Large and } \\
\text { middlow- } \\
\text { dimension }\end{array}$ & $\begin{array}{c}\text { Mostly used } \\
\text { for } \\
\text { spatial }\end{array}$ \\
\hline $\mathrm{GCHL}$ & 2005 & Grid & $\begin{array}{l}\text { Eulidean } \\
\text { distance }\end{array}$ & 2 & In-Sensitive & $\begin{array}{c}\text { Arbitrary } \\
\text { shape }\end{array}$ & $\begin{array}{l}\text { Oversize } \\
\text { high- } \\
\text { dimension }\end{array}$ & $\begin{array}{l}\text { Information } \\
\text { processing }\end{array}$ \\
\hline ACODF & 2004 & Others & $\begin{array}{l}\text { Eulidean } \\
\text { distance }\end{array}$ & 1 & - & $\begin{array}{c}\text { Sphere, } \\
\text { non-sphere }\end{array}$ & $\begin{array}{l}\text { Small, } \\
\text { high- } \\
\text { dimension }\end{array}$ & $\begin{array}{c}\text { Get optimal } \\
\text { value } \\
\text { fast }\end{array}$ \\
\hline
\end{tabular}

Combining both algorithms in references and the methods proposed in this paper, we can make the conclusion that: clustering algorithms and the results are unpredictable, in practical researches, we should choose appropriate clustering algorithms to solve different kind of data problems in order to obtain the best clustering results.

Volume 4 Issue 12, December 2015 


\section{International Journal of Science and Research (IJSR) \\ ISSN (Online): 2319-7064}

Index Copernicus Value (2013): 6.14 | Impact Factor (2014): 5.611

\section{References}

[1] Jain AK, Flynn PJ. Image segmentation using clustering. In: Ahuja $\mathrm{N}$, Bowyer $\mathrm{K}$, eds. Advances in Image Understanding: A Festchrift for Azriel Rosenfeld. Piscataway: IEEE Press, 1996. 65-83.

[2] Cades I, Smyth P, Mannila H. Probabilistic modeling of transactional data with applications to profiling, visualization and prediction, sigmod. In: Proc. of the 7th ACM SIGKDD. San Francisco: ACM Press, 2001. 37-46.

[3] Jain AK, Murty MN, Flynn PJ. Data clustering: A review. ACM Computing Surveys, 1999, 31(3): 264-323.

[4] Gelbard R, Goldman O, Spiegler I. Investigating diversity of clustering methods: An empirical comparison. Data \& Knowledge Engineering, 2007, 63(1): 155-166.

[5] Jain AK, Dubes RC. Algorithms for Clustering Data. Prentice-Hall Advanced Reference Series, 1998. 1-334.

[6] Jain AK, Duin RPW, Mao JC. Statistical pattern recognition: A review. IEEE Trans. on Pattern Analysis and Machine Intelligence, 2000, 22(1): 4-37.

[7] Sambasivam S, Theodosopoulos N. Advanced data clustering methods of mining Web documents. Issues in Informing Science and Information Technology, 2006, (3): 563-579.

[8] Marques JP, Written; Wu YF, Trans. Pattern Recognition Concepts, Methods and Applications. 2nd ed., Beijing: Tsinghua University Press, 2002. 51-74.

[9] Gelbard R, Spiegler I. Hempel's raven paradox: A positive approach to cluster analysis. Computers and Operations Research, 2000, 27(4): 305-320.

[10]Zhang B, Srihari SN. Properties of binary vector dissimilarity measures. In: Proc. of the JCIS CVPRIP 2003. 2003. 26-30.

[11]Kumar P, Krishna PR, Bapi RS, De SK. Rough clustering of sequential data. Data \& Knowledge Engineering, 2007, 3(2): 183-199.

[12]Huang Z. A fast clustering algorithm to cluster very large categorical data sets in data mining. In: Proc. of the SIGMOD Workshop on Research Issues on Data Mining and Knowledge Discovery. Tuscon, 1997. 146-151.

[13]Huang Z. Extensions to the $k$-means algorithm for clustering large data sets with categorical values. Data Mining and Knowledge, Discovery II, 1998, (2): 283-304.

[14]Huang Z, Ng MA. Fuzzy $k$-modes algorithm for clustering categorical data. IEEE Trans. on Fuzzy Systems, 1999, 7(4): 446-452.

[15] Chaturvedi AD, Green PE, Carroll JD. $K$-modes clustering. Journal of Classification, 2001, 18(1): 35-56.

[16] Goodman LA. Exploratory latent structure analysis using both identifiable and unidentifiable models. Biometrika, 1974, 61(2): 215-231.

[17] Huang ZX, Michael K. A note on $K$-modes clustering. Journal of Classification, 2003, 20(2): 257-261.

[18] Sun Y, Zhu QM, Chen ZX. An iterative initial-points refinement algorithm for categorical data clustering. Pattern Recognition Letters, 2002, 23(7): 875-884.

[19] Bradley PS, Fayyad UM. Refining initial points for $k$-means clustering. In: Proc. of the 15 th Internet Conf. on Machine Learning. San Francisco: Morgan Kaufmann Publishers, 1998. 91-99.

[20]Ding C, He X. K-Nearest-Neighbor in data clustering: Incorporating local information info global optimization. In: Proc. of the ACM Symp. on Applied Computing. Nicosia: ACM Press, 2004. 584-589.

[21] Lyer NS, Kandel A, Schneider M. Feature-Based fuzzy classification for interpretation of mammograms. Fuzzy Sets System, 2000, 114(2): 271-280.

[22] Yang MS, Hu YJ, Lin KCR, Lin CCL. Segmentation techniques for tissue differentiation in MRI of ophthalmology using fuzzy clustering algorithm. Journal of Magnetic Resonance Imaging, 2002, (20): 173-179.

[23] Li J, Gao XB, Jiao LC. A new feature weighted fuzzy clustering algorithm. ACTA Electronic Sinica, 2006,34(1): 412-420.

[24] Kononenko I. Estimating attributes: Analysis and extensions of relief. In: Proc, of the 17th European Conf. ON Machine Learning. LNCS 784, 1994. 171-181.

[25] Cai WL, Chen SC, Zhang DQ. Fast and robust fuzzy $c$-means clustering algorithms incorporating local information for image segmentation. Pattern Recognition, 2007, 40(3): 825-833.

[26] Harel D, Koren Y. Clustering spatial data using random walks. In: Proc. of the 7th ACM SIGKDD Int'l Conf. Knowledge Discovery and Data Mining. New York: ACM Press, 2001. 281-286.

[27] Karypis G, Han EH, Kumar V. CHANELEON: A hierarchical clustering algorithm using dynamic modeling. IEEE Computer, 1999, 2(8): 68-75.

[28] Estivill-Castro V, Lee I. AUTOCLUST: Automatic clustering via boundary extraction for mining massive point-data sets. In Abrahart J, Carlisle BH, eds. Proc. of the 5th Int'l Conf. on Geocomputation. 2000. 23-25.

[29] Li YJ. A clustering algorithm based on maximal $\theta$-distant subtrees. Pattern Recognition, 2007, 40(5): 1425-1431.

[30]Zhao YC, Song J. GDILC: A grid-based density isoline clustering algorithm. In: Zhong YX, Cui S, Yang Y, eds. Proc. of the Internet Conf. on Info-Net. Beijing: IEEE Press, 2001. 140-145.

[31] Ma WM, Chow E, Tommy WS. A new shifting grid clustering algorithm. Pattern Recognition, 2004, 37(3): 503-514.

[32] Pilevar AH, Sukumar M. GCHL: A grid-clustering algorithm for high-dimensional very large spatial data bases. Pattern Recognition Letters, 2005, 26(7): 999-1010

[33] Nanni M, Pedreschi D. Time-Focused clustering of trajectories of moving objects. Journal of Intelligent Information Systems, 2006,27(3): 267-289.

[34]Birant D, Kut A. ST-DBSCAN: An algorithm for clustering spatial-temporal data. Data \& Knowledge Engineering, 2007, 60(1): 208-221.

\section{Volume 4 Issue 12, December 2015}




\section{International Journal of Science and Research (IJSR) \\ ISSN (Online): 2319-7064}

Index Copernicus Value (2013): 6.14 | Impact Factor (2014): 5.611

[35] Tsai CF, Tsai CW, Wu HC, Yang T. ACODF: A novel data clustering approach for data mining in large databases. Journal of Systems and Software, 2004, 73(1):133-145.

Volume 4 Issue 12, December 2015 www.ijsr.net 